\title{
Equilibration in Quark Gluon Plasma
}

\author{
Santosh K Das, Jan-e Alam and Payal Mohanty \\ Variable Energy Cyclotron Centre, 1/AF, Bidhan Nagar, Kolkata 700064, India
}

\begin{abstract}
The hydrodynamic expansion rate of quark gluon plasma (QGP) is evaluated and compared with the scattering rate of quarks and gluons within the system. Partonic scattering rates evaluated within the ambit of perturbative Quantum Choromodynamics (pQCD) are found to be smaller than the expansion rate evaluated with ideal equation of state (EoS) for the QGP. This indicate that during the space-time evolution the system remains out of equilibrium. Enhancement of pQCD cross sections and a more realistic EoS keep the partons closer to the equilibrium.
\end{abstract}

\section{INTRODUCTION}

The main aim of nuclear collisions at the Relativistic Heavy Ion Collider (RHIC) and the Large Hadron Collider (LHC) energies is to create a thermalized system of quarks and gluons - mimicking the situation that prevailed after few microsecond of the big bang. The expansion time scale in the microsecond old universe is much larger than the interaction time scale of quarks and gluons. Consequently during the evolution the system maintains equilibrium. In the case of heavy ion collisions (HIC), however, these time scales have comparable magnitude. For HIC the two pertinent issues for the equilibration are (i) do the quarks and gluons achieve equilibrium and (ii) in case they do, can the equilibrium be maintained during the evolution ? or what kinds of EoS and interaction cross sections are required for the maintenance of equilibration. Some of these issues will be addressed in the present work.

\section{THERMAL EQUILIBRIUM IN HEAVY ION COLLISIONS}

To address the first issue the time evolution of average momentum $\left.\left(<p_{T}\right\rangle\right)$ of the quarks and gluons have been studied and compare with its equilibrium value. In relaxation time approximation it can be shown that the evolution of the $\left\langle p_{T}\right\rangle$ is governed by the equation:

$$
\frac{d<p_{T}>}{d \tau}=-\frac{<p_{T}>-<p_{T \mathrm{e} q}>}{\tau_{\text {relax }}}
$$

$\tau_{\text {relax }}$ is the relaxation time evaluated with $\mathrm{pQCD}$ cross section for $\alpha_{s}=0.3, p_{T \mathrm{e} q}$ is the value of $p_{T}$ in equilibrium. The equilibrium time, $\tau_{\mathrm{e} q}$ is obtained from the condition: $\left\langle p_{T}\right\rangle \rightarrow p_{T \mathrm{e} q}$ as $\tau \rightarrow \tau_{\mathrm{e} q}$. It has been found that the value $\tau_{\mathrm{e} q}$ depends weakly on initial value of $\left\langle p_{T}\right\rangle$.

To address the second issue we assume that the quarks and gluons produced initially in thermal equilibrium and check whether it can maintain the equilibrium during the entire evolution processes by comparing their scattering rates with the expansion rate of the matter. The scattering time scales $\left(\tau_{\text {scatt }}\right)$ of various partons are evaluated in pQCD and these time scales are compared with the expansion time scale $\left(\tau_{\mathrm{e} x p}\right)$. For maintenance of thermal equilibrium the following criteria should be satisfied:

$$
\tau_{\mathrm{e} x p} \geq \alpha \tau_{\text {scatt }}
$$

where $\alpha \sim O(1)$, is a constant. The criteria given in Eq. 2 is reverse to the one used to study the freeze-out of various species of particles during the evolution of the early universe [1]. Similar condition is used in heavy ion collisions also to study freeze-out of hadrons [2]. Physically Eq. 2] means that the time taken by a partons to travel a distance of one mean free path by their thermal 
motion they have collectively receded from each other by less than one mean free path 2]. The $\tau_{\text {scatt }}$ is determined for parton types $i$ by the expression

$$
\tau_{\text {scatt }}^{i}=\left(\sum \sigma_{i j} v_{i j} n_{j}\right)^{-1}
$$

where $\sigma_{\mathrm{i} j}$ is the total cross section for particles $i$ and $j, v_{i j}$ is the relative velocity between $i$ and $j$ and $n_{j}$ is the density of the particle type $j$ in the medium.

The scattering time scales for partons have been calculated by taking into account the following leading order processes $g g \rightarrow g g, g g \rightarrow q \bar{q}, q(\bar{q}) g \rightarrow q(\bar{q}) g, q q \rightarrow q q, q \bar{q} \rightarrow q \bar{q}$ for light flavours and gluons [3]. Here $q$ stands for light quarks and $g$ denotes gluons. For evaluating $\tau_{\text {scatt }}$ for heavy quarks $(Q)$ the $\mathrm{pQCD}$ processes are taken from [4]. The infrared divergence appearing in case of massless particle exchange in the $t$-channel has been shielded by Debye mass.

The expansion time scale can be defined as:

$$
\tau_{\mathrm{exp}}^{-1}=\frac{1}{\epsilon(\tau, r)} \frac{d \epsilon(\tau, r)}{d \tau}
$$

where $\epsilon(\tau, r)$ is the energy density at a proper time $\tau$ and radial co-ordinate $r . \epsilon(\tau, r)$ is obtained by solving the hydrodynamical equation:

$$
\partial_{\mu} T^{\mu \nu}=0
$$

with the assumption of boost invariance along longitudinal direction [5] and cylindrical symmetry of the system [6]. In Eq. (5), $T^{\mu \nu}=(\epsilon+P) u^{\mu} u^{\nu}-g^{\mu \nu} P$ is the energy momentum tensor, $P$ is the pressure, $u^{\mu}$ denotes four velocity and $g^{\mu \nu}$ stands for metric tensor. We consider a net baryon free QGP here, therefore the baryonic chemical potential $\left(\mu_{B}\right)$ is zero.

\section{INITIAL CONDITIONS AND EOS}

The expansion rates for RHIC and LHC energies have been calculated using the initial conditions, $T_{i}=400 \mathrm{MeV}, \tau_{i}=0.2 \mathrm{fm}$ for RHIC which gives $d N / d y \sim 1100$ and $T_{i}=700 \mathrm{MeV}, \tau_{i}=0.08 \mathrm{fm}$ for LHC giving $d N / d y=2100$ [7]. The initial radial velocity has been taken as zero. Two sets of equation of state (EoS) have been used to study the sensitivity of the results on EoS. SET-I: In a first order phase transition scenario - we use the bag model EOS for the QGP phase and for the hadronic phase all the resonances with mass $\leq 2.5 \mathrm{GeV}$ have been considered [8]. and SET-II: The EOS is taken from lattice QCD (1QCD) calculations performed by the MILC collaboration [9].

\section{RESULTS}

The time evolution of $\left\langle p_{T}>\right.$ obtained by solving Eq. 1 is depicted in Fig. 1 for a system of quarks and gluons. The results with pQCD interactions indicate that the quarks are unlikely thermalize both at RHIC and LHC energies. The possibility of gluon thermalization at LHC is very strong. The gluon thermalization time scale seems to comparable to the life time of the QGP at RHIC energies indicating that the gluons may remain out of equilibrium during the evolution. However, any non-perturbative effects may drive the system faster toward equilibrium.

In Fig 2 the scattering time scale is contrasted with the expansion time scale for RHIC energy for two types of EoS mentioned above. Expansion rate for both the EoS is similar at early time but differ little at the late stage of the evolution. For the sake of comparison the expansion rate for the extreme case of free streaming is also displayed. The scattering rates are evaluated with pQCD cross sections (left panel). The condition for equilibration in Eq. 22 indicates that the gluons 

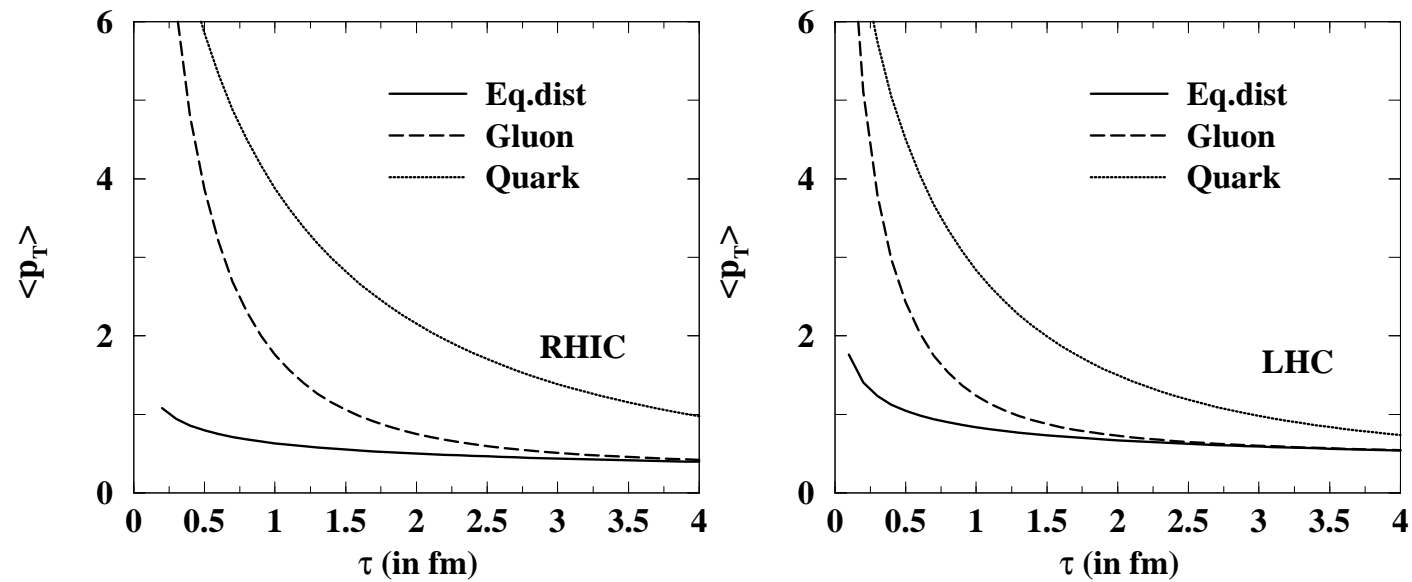

FIG. 1: Evolution of partonic average momentum with time for a non-expanding system for RHIC and LHC energies.

remains close to equilibrium, however the charm and bottom (not shown in the figure) quarks remain out of equilibrium during the entire evolution history.

The analysis of the experimental data within the ambit of relativistic hydrodynamics suggest that the matter formed in $\mathrm{Au}+\mathrm{Au}$ collisions at RHIC achieve thermalization. One possible reasons for the thermalization to occur is that the partons interact strongly after their formation. It is argued in [10] that the onset of thermalization in the system formed in heavy ion collisions at relativistic energies can not be achieved without non-perturbative effects. It has also been shown in [11] that a large enhancement of the pQCD cross section is required for the reproduction of experimental data on elliptic flow at RHIC energies. Therefore, the pQCD cross sections used to derive the results shown in Fig. 2 (left panel) should include non-perturbative effects. To implement this we enhance the pQCD cross sections by a factor of 2 . The resulting scattering time is compared with the expansion time in Fig. 2 (right panel). It is observed that the gluons are kept in equilibrium throughout the evolution, light quarks are closer to the equilibrium as compared to the heavy flavours.

In Figs. 3 the results for LHC are displayed for the two time scales mentioned above for pQCD and enhanced cross sections. The expansion becomes faster at LHC than RHIC because of the higher internal pressure. As a consequence, it is interesting to note that the thermalization scenario at LHC does not differ drastically from RHIC.

\section{SUMMARY}

We have evaluated the rate of scattering of various partons in QGP and compared the scattering time scale with the expansion time scale to examine whether the evolving mater is in thermal equilibrium or not. It is found that gluons remain close to equilibrium throughout the evolution. Quarks gets equilibrated at the late stage of the evolution both for RHIC and LHC energies. Enhancement of pQCD cross sections and realistic lQCD EoS drives the system toward equilibrium faster.

\section{References}

[1] E. W. Kolb and M. S. Turner, The Early Universe, Addison-Wesley Pub. Co., NY, 1990. 

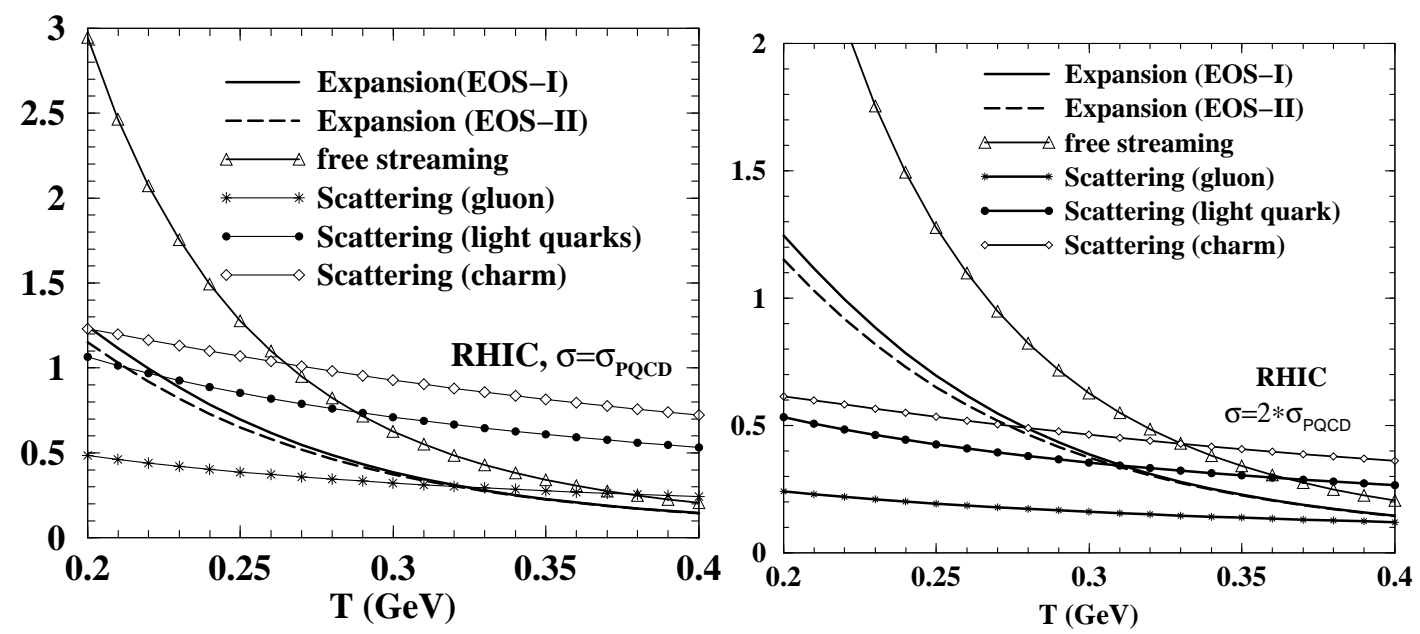

FIG. 2: Expansion versus scattering time scale for RHIC energy at $r=1 \mathrm{fm}$.
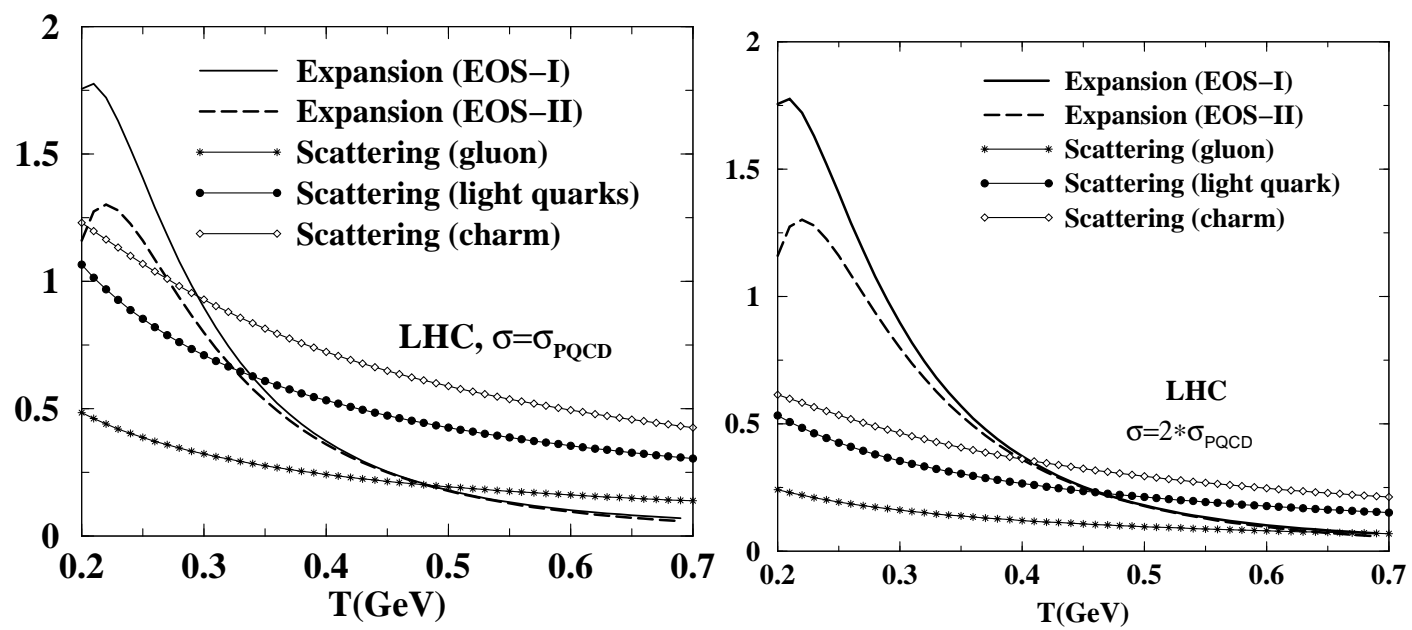

FIG. 3: Expansion versus scattering time scale for LHC energy at $r=1 \mathrm{fm}$.

[2] F. S. Navarra et al., Phys. Rev. C 45, 2552 (1992).

[3] R. D. Field, Application of Perturbative QCD, Addison-Wesley Pub. Co., NY, 1989.

[4] B.L. Combridge, Nucl.Phys.B 151, 429 (1979).

[5] J. D. Bjorken, Phys. Rev. D 27, 140 (1983).

[6] H. von Gersdorff et al., Phys. Rev. D 34, 794 (1986).

[7] N. Armesto et al.(eds.), J. Phys. G: Nucl. Part. Phys. 35, 054001 (2008).

[8] B. Mohanty and J. Alam Phys. Rev. C 68, 064903 (2003).

[9] C. Bernard et al., Phys. Rev. D 75, 094505 (2007).

[10] Y. V. Kovchegov, Nucl. Phys. A. 762, 298 (2005).

[11] D. Molnar and M. Gyulassy, Nucl. Phys. A 697, 495 (2002). 